\title{
El rol del estudiante en el fenómeno del Compromiso Académico en Educación Superior ${ }^{1}$
}

\author{
The role of the student in the phenomenon of Study Engagement in Higher Education
}

\author{
Jorge Ignacio Maluenda Albornoz ${ }^{2}$ \\ Felipe Moraga Villablanca ${ }^{3}$ \\ Alejandro Díaz Mujica ${ }^{4}$
}

\begin{abstract}
RESUMEN
El gran interés surgido frente al estudio del Compromiso Académico, debido a su vinculación con el desempeño y el abandono de los estudios universitarios, ha traído como consecuencia la existencia de una investigación prolífera y distintas aproximaciones. El objetivo de este trabajo es analizar las diferencias conceptuales y/o metodológicas entre dos aproximaciones al fenómeno provenientes desde la Teoría de la Autodeterminación. Se realiza un ensayo que analiza críticamente las principales líneas de trabajo, las teorías, los conceptos y sus modelos explicativos, así como los potenciales efectos sobre su desarrollo. Se concluye la posibilidad de que, la concepción del Compromiso Académico opere desde un enfoque topdown donde la construcción científica de la teoría, modelos teóricos y conceptos afecte la práctica cotidiana, los significados y marcos de funcionamiento en la educación. Las conceptualizaciones predominantes sobre el Compromiso Académico podrían estar favoreciendo un rol pasivo del estudiante, además de incrementar la responsabilidad de los sistemas educativos sobre la producción de entornos que puedan favorecerlo.

Palabras clave: Compromiso académico, Estudiantes universitarios, Teoría de la autodeterminación, Modelo de recursos bajo demanda, Rol del estudiante.
\end{abstract}

\begin{abstract}
The great interest that emerged from the study of the Study Engagement, due to its connection with the achievement and the abandonment of university studies, has resulted in the existence of prolific research and different approaches. The objective of this work is to analyze the conceptual and methodological differences between two approaches to this phenomenon based on Self-determination Theory. An essay is carried out that analyzes the main lines of work, theories, concepts and their explanatory models, as well as the potential effects on their development. It concludes the possibility that, the conception of the Study Engagement operates from a top-down approach where the scientific construction of the theory, theoretical models and concepts affect the daily practice, the meanings and frameworks of functioning in education. The predominant conceptualizations about the Academic Commitment could be favoring a passive role of the student, in addition to increasing the responsibility of the educational systems on the production of environments that can favor it.
\end{abstract}

Key Words: Study Engagement, University students, Self-Determination Theory, Job DemandsResourses, Student's role.

\footnotetext{
1 "Proyecto FONDECYT 1161502 Modelo explicativo de la permanencia y el abandono de los estudios universitarios, basado en procesos cognitivo motivacionales".

${ }^{2}$ UdeC. Magíster en Política y Gobierno. Doctorando en Psicología. Chile.

Correo electrónico: Jorgemaluenda@udec.cl

${ }^{3}$ UdeC. Magíster en Psicología. Centro de apoyo al estudiante. Chile.

Correo electrónico: femoraga@udec.cl

${ }^{4}$ UdeC. Doctor en Psicología. Departamento de Psicología. Chile. Correo electrónico: adiazm@udec.cl
}

Recepción: 21/1/2019 Aceptación: 19/2/2019. 


\section{Introducción}

En la actualidad existe preocupación e interés en la Educación Superior por descifrar las variables del proceso educativo que permitan predecir de mejor manera el desempeño de los estudiantes, su permanencia y el alcance de los logros académicos esperados. A la vez, se busca contrarrestar los efectos del abandono y el fracaso académico (Gómez et al., 2015; Pérez-villalobos et al., 2012).

El panorama para América Latina y el Caribe es complejo. La matrícula se ha expandido de un $21 \%$ a un $43 \%$ entre los años 2000 y 2013 con una tasa de retención de apenas un 46\%. Esto implica, según cifras del Banco Mundial (2017) que un 22\% aproximado de la población entre 25 y 29 años ha abandonado sus estudios (Ferreyra, Avitabile, Botero, Haimovich, \& Urzúa, 2017), trayendo como consecuencia costos económicos para la familia/estudiante y para el estado, gravámenes sociales y psicológicos de distinta índole, entre otros.

Debido a lo anterior, existe un alto grado de interés en el estudio del Compromiso Académico por la relaciones encontradas en diversos estudios entre este constructo y el desempeño (Cox, Bjornsen, \& Krieshok, 2015; Gómez et al., 2015; Salanova, Schaufeli, Martínez, \& Bresó, 2009; Schaufeli, Martínez, Marques, Salanova, \& Bakker, 2002), y de manera inversa con el abandono de los estudios (Archambault, Janosz, Fallu, \& Pagani, 2009; Casuso-holgado et al., 2013; Chang, Sharkness, Hurtado, \& Newman, 2014; Cox et al., 2015; Hu \& McCormick, 2012). Además, este fenómeno ha sido considerado importante desde la academia y la política pública para abordar los problemas relacionados con el bajo logro académico y las altas tasas de aburrimiento, alienación y abandono existentes (Fredricks, Blumenfeld, \& Paris, 2004).

A pesar de esta clara orientación, se han observado resultados en algunas investigaciones que reflejan contraposiciones a la corriente principal de hallazgos (Casuso-holgado et al., 2013; Salanova et al., 2009). Estas diferencias podrían estar vinculadas con el amplio espectro de formas en que se ha conceptualizado el Compromiso Académico (Fredricks \& Mccolskey, 2012), los distintos tipos de participantes que han involucrado los estudios (estudiantes talentosos y en riesgo), las diversas formas de evaluación no equivalentes (centradas en una o más dimensiones) y basadas en distintas técnicas (autorreporte, la observación conductual, etc.), entre otros factores (Fredricks et al., 2004). 


\section{Objetivo}

El presente ensayo analiza las diferencias conceptuales entre dos aproximaciones al fenómeno del Compromiso Académico provenientes desde la Teoría de la Autodeterminación. Esto a partir de un análisis de sus bases conceptuales e implicaciones en el rol del estudiante en relación con su contexto. Por último, se propone que estas aproximaciones involucran concepciones epistemológicas específicas sobre el estudiante que juegan un rol en el proceso educativo en el contexto de la Educación Superior.

\section{Discusión}

\subsection{Dos grandes líneas de investigación del Compromiso Académico}

El estudio del Compromiso Académico se ha desarrollado, principalmente, desde dos líneas de investigación que organizan la distinta investigación en la temática: una conceptualización que lo comprende como un meta-constructo tridimensional, otra, que lo concibe como constructo cognitivo-afectivo.

La primera línea de investigación, desarrollada principalmente en el contexto escolar y basada en la Teoría de la Autodeterminación (TAD), propone la integración entre tres formas unidimensionales de comprensión del fenómeno (Compromiso Conductual, Emocional y Cognitivo) en la forma de un meta-constructo que reconoce la multidimensionalidad e interrelación entre sus sub-dimensiones (Fredricks et al., 2004). Esta perspectiva de trabajo supone que los estudiantes tienen tres necesidades motivacionales fundamentales: autonomía, competencia y relación (Deci, Olafsen, \& Ryan, 2017).

La Autonomía se satisface cuando el estudiante siente que toma opciones y se motiva por factores intrínsecos más que externos, la Competencia se favorece cuando la estructura de la clase permite alcanzar los resultados deseados y la Relación ocurre cuando profesores y pares crean un entorno de apoyo y preocupación (Fredricks \& Mccolskey, 2012).

Se supone que una institución educativa que provea a los estudiantes oportunidades para abordar estas tres necesidades conducirán a mayores niveles de Compromiso Académico (Fredricks \& Mccolskey, 2012). Por lo tanto, el esfuerzo, persistencia y conducta prosocial en clases (Compromiso Conductual), el alto interés, alto entusiasmo, baja ansiedad y bajo aburrimiento (Compromiso Emocional), la 
concentración, pensamiento estratégico, estrategias de aprendizaje sofisticado y autorregulación (Compromiso Cognitivo) (Fredricks et al., 2004; Veiga, Reeve, Wentzel, $\&$ Robu, 2014) y las acciones intencionales de agencia para enriquecer la experiencia de aprendizaje de una materia o de la escuela (Compromiso de Agencia) (Veiga et al., 2014) surgen producto de la interacción del individuo con su entorno para la satisfacción de estas distintas necesidades.

Desde esta aproximación, el fenómeno es entendido en términos de las acciones, o manifestaciones conductuales, emocionales y cognitivas de la motivación (Skinner, Furrer, Marchand, \& Kindermann, 2008), donde la motivación es necesaria pero no suficiente como precursora del Compromiso (Appleton, Christenson, \& Furlong, 2008), y donde la institución de educación y sus diversos componentes juegan un rol fundamental en su surgimiento.

La segunda aproximación al Compromiso Académico proviene de la Salud Ocupacional, basada en el Modelo de Recursos Bajo Demanda (JD-R) que también encuentra sus raíces en la TAD. Este modelo es transferido al contexto académico por su similitud con el contexto laboral en tanto que, la actividad estudiantil contempla al igual que la laboral, actividades coercitivas y objetivos específicos que alcanzar (Salanova et al., 2009).

La explicación desde el Modelo JD-R sugiere que el Compromiso con el trabajo resultaría de la tensión existente entre las demandas laborales y los recursos disponibles por el trabajador para hacer frente a ellas, donde contar con los recursos necesarios para abordar las demandas enfrentadas favorecería el Compromiso y motivación con el trabajo, y lo inverso, generaría efectos perniciosos sobre estos factores y la salud (Bakker \& Demerouti, 2014).

Los recursos laborales comprenden los aspectos del trabajo que son funcionales para alcanzar metas del trabajo, reducir las demandas del trabajo, y estimular el crecimiento y desarrollo personal (Ej. Retroalimentación del desempeño, control del trabajo y apoyo social de los colegas), mientras que, los recursos personales, se definen como los aspectos del Self que están asociados con la resiliencia y que refieren a la habilidad para controlar e impactar el entorno con éxito (Ej. Autoeficacia, optimismo y estabilidad emocional) (Schaufeli, 2013).

Las demandas laborales, por su parte, hacen referencia a aquellos aspectos físicos, psicológicos, organizacionales o sociales del trabajo que requieren un esfuerzo sostenido 
y conllevan costes fisiológicos y psíquicos (Ej. Presión laboral, exigencias de clientes, etc.) (Bakker \& Demerouti, 2014).

El Compromiso Académico desde esta perspectiva es entendido como estado cognitivo-afectivo, distinto pero correlacionado negativamente con el Burnout (Schaufeli, 2013), que se caracteriza por el vigor, la dedicación a los propios estudios y el ser absorbido por las tareas relacionadas con este (Schaufeli, Salanova, González-Romá, \& Bakker, 2002) y que se produce en la interacción entre demandas y recursos antes señalada, la cual, es transferible al contexto del desenvolvimiento académico de los estudiantes (Salanova et al., 2009).

\subsection{Nociones sobre el rol del estudiante en el Compromiso Académico}

Las propuestas de comprensión del Compromiso Académico anteriormente presentadas implican una raíz teórica común (TAD) con variaciones en los modelos explicativos y las aproximaciones metodológicas. En ambos casos se otorga un rol muy importante a las contingencias externas que, en interacción con características intrínsecas del individuo desembocan en un estado de Compromiso Académico determinado.

El Modelo JD-R realza una focalización en la tarea y propone la generación del Compromiso Académico como una consecuencia de la orientación funcional de los individuos frente a las demandas (tareas, exigencias, metas, etc.). En este sentido, pierden preponderancia aspectos relacionales incluidos en la vida académica como son el valor intrínseco de las relaciones interpersonales y aspectos vinculados con la autorrealización de los estudiantes que van más allá de su valor funcional vinculado al estudio (amistad, admiración, contribución social, vocación, etc.). Por lo tanto, queda supeditada la comprensión del fenómeno a la observación del vínculo entre el estudiante y sus tareas.

En el caso de la perspectiva Tridimensional cobran gran relevancia tanto los factores relacionales (relaciones con los profesores y pares) como aquellos de administración del aula y la institución (estructura de clases, estrategias de enseñanza, etc.). Sin embargo, a diferencia del enfoque anterior, se considera explícitamente la participación del estudiante en el proceso. Es así que la propia toma de decisiones y las motivaciones personales forman parte de las formulaciones desde esta perspectiva. En este punto, el modelo JD-R propone que los estudiantes serían sujetos reactivos frente a 
sus labores, y por lo tanto, su rol es mayoritariamente pasivo en la construcción de su Compromiso Académico.

El estado cognitivo-afectivo que supone el Compromiso Académico es entonces un resultado de diversos factores a los que el estudiante está sometido, donde su participación ocurre en el enfrentamiento de los desafíos académicos, más no en el desarrollo de un propio camino educativo que lleve a la construcción de un perfil, identidad profesional y/o futuro profesional al que se aspira.

En el caso del modelo Tridimensional, a pesar de señalar explícitamente la participación activa del estudiante, no es claro su rol ni tampoco el monto o magnitud de esta participación respecto de los aspectos contextuales.

El estudiante participaría desde sus propias motivaciones en la toma de decisiones pero, al igual que en el contexto educativo en general, se deja entrever una inclinación por una mayor preponderancia de las “condiciones" en que el estudiante está inscrito para impulsar su grado de Compromiso Académico. Además, pareciera que las motivaciones y toma de decisiones consideradas se circunscriben al "universo educativo más cotidiano" por sobre una orientación que reconoce la participación activa del estudiante en la construcción de proyecciones vitales, relacionadas con la identidad y con su futuro.

En particular, en este caso, puede ser que el desarrollo de este enfoque en el contexto escolar explique esta aparente tendencia, donde se supone que los estudiantes tienen menos capacidad para el funcionamiento autónomo - que desarrollan de manera progresiva a través de la escolaridad - y por lo tanto, un fuerte rol de asistencia y dirección educativa podría justificarse

Con todo, no es posible determinar con mayor precisión sobre este último punto, debido a la falta de desarrollo teórico de esta perspectiva en el contexto de la Educación Superior.

Un punto que parece trascendental en este análisis se refiere fundamentalmente a las necesidades base contempladas en la TAD (Deci et al., 2017) que son el fundamento de las dos grandes líneas de trabajo antes indicadas. Tal como se ha señalado, la TAD propone como principales necesidades que guían la comprensión de la conducta humana las de Autonomía, Competencia y Relación (Ryan \& Deci, 2000). Vale la pena preguntarse por el ajuste de este modelo de comprensión de la conducta en el concierto del desarrollo del Compromiso Académico de los estudiantes. 
El planteamiento que se propone en este ensayo es que, si bien existe evidencia científica que respalda el valor explicativo de estas necesidades en la mayor parte del comportamiento humano, es posible también que estas no sean las únicas ni las más importantes para la comprensión del Compromiso Académico.

Se observa que estas necesidades se ajustan de manera importante en la comprensión del comportamiento de los estudiantes en torno a su desenvolvimiento más cotidiano. Por ejemplo, la satisfacción de la necesidad de relación favorecería el sentirse parte de un curso, o considerarse valorado por sus profesores. La satisfacción de la necesidad de autonomía y de competencia, por su parte, podría contribuir al desarrollo de la autoeficacia y de un buen rendimiento, aspectos que podrían contribuir al Compromiso. Sin embargo, pareciera ser que no permite explicar factores relacionados con motivaciones trascendentales como son las aspiraciones y proyecciones vitales, o las de tipo altruista como son los deseos de contribuir al desarrollo de la disciplina que se estudia, a los destinatarios de la futura práctica laboral y a la sociedad en su conjunto.

Estos aspectos pueden tener un importante rol en el desarrollo del Compromiso Académico, sobretodo, cuando existe investigación que ha encontrado participación más activa, mayor y mejor aprendizaje cuando se trabaja con orientación altruista (Bielefeldt \& Canney, 2014; Gandhi, Maalouf, \& Patton, 2015; Knizley \& Coleman, 2014; Markus, Howard, \& King, 1993) y cuando se valora la utilidad de lo aprendido para el futuro ejercicio del rol profesional (Navarro, Vaccarezza, González, \& Catalán, 2015).

\section{3 ¿Qué comprensión del Compromiso Académico estamos sosteniendo?}

Un primer aspecto interesante que plantear es la pregunta acerca de cómo las conceptualizaciones del Compromiso Académico hoy imperantes dan cuenta del proceso dialéctico en que, estudiantes, profesores y otros actores del proceso educativo participan.

De acuerdo con Blumer, los fenómenos se construyen en la interacción social y estos se re-articulan en torno a las prácticas y significados que los individuos les atribuyen (Carvalho, De Oliveira, \& Pereira do Rêgo, 2010), por lo que vale la pena analizar críticamente esta cuestión, considerando las improntas ya descritas para cada uno de las aproximaciones predominantes al Compromiso Académico. 
¿Son capaces estas conceptualizaciones de representar las formas en cómo los participantes de las diversas comunidades de aprendizaje entienden y experimentan el Compromiso Académico? Aparejado a esta interrogante surge como otra cuestión de interés capital, la reflexión acerca de la influencia del concepto científicamente construido sobre las prácticas efectivas de los distintos actores del proceso educativo.

Siguiendo a Austin, el lenguaje tiene una capacidad performativa que puede producir no solo códigos de comprensión comunes, sino también significados que afecten el comportamiento (Ramírez, 2015), en este caso, de los partícipes del proceso educativo. De este modo, los planteamientos ofrecidos desde la teoría y vertidos en las investigaciones podrían impactar de manera sustancial en la micro-cultura educativa produciendo entramados de significados acorde a los mismos.

De acuerdo a Blumer las personas se comportan de acuerdo al significado que atribuyen a las cosas (Carvalho et al., 2010) por lo tanto, la definición sobre qué significa ser una estudiante comprometido y cuáles son las explicaciones a este tipo de comportamientos adquiere una connotación no menos importante.

Con todo, si la construcción del concepto de Compromiso Académico está ejerciendo una influencia top-down sobre el fenómeno habría que también considerar los efectos de esta influencia. En este caso, la construcción conceptual sostenida académicamente proveería el marco de referencia y los límites de entendimiento que impulsen las prácticas educativas a través de las expectativas, estándares, incentivos, políticas públicas, entre otros mecanismos. Por ejemplo, si la concepción científica del Compromiso Académico configura al estudiante como agente pasivo en la ocurrencia del fenómeno y/o como objeto - y no sujeto - del proceso educativo, el desarrollo de estas nociones puede estar generando una compleja estructura de sentido, incentivos y prácticas que fortalecen dicha orientación. Del mismo modo, una orientación de corto plazo y meramente funcional del Compromiso Académico (orientada a metas y logros de rendimiento) puede coartar los motivos y aspiraciones de los estudiantes y de todo el sistema educativo. Además, puede contribuir a la alineación de los fines que persigue el estudiante, dejando de favorecer e incluso coartando la posibilidad desarrollo de propósitos de formación personal con orientaciones altruistas.

De acuerdo a Berger y Luckman (Rizo, 2015) las tipificaciones sociales producen instituciones sociales determinadas las que, a su vez, afectan los comportamientos de los individuos 
Las concepciones construidas desde la academia podrían ser replicadas en los niveles prácticos de la Educación Superior (en la enseñanza y práctica cotidiana) a partir de las instituciones construidas. Con ello, tanto las elaboraciones teóricas y conceptuales que realizamos acerca del Compromiso Académico como sus definiciones operacionales - materializadas comúnmente en instrumentos de medida, políticas institucionales, guías prácticas, herramientas de gestión, etc. - pueden transformarse en orientaciones de comportamiento con suficiente peso como para delimitar e impulsar determinados marcos de acción. Por ejemplo, describir el Compromiso Académico Conductual como "seguir las reglas", "ajustarse a la clase" y "evitar conductas incorrectas" no está exento de una visión paradigmática sobre la educación y de un poder sobre el comportamiento de los estudiantes, muy distinto de "involucrarse en la cultura educativa" o "participar de un gobierno estudiantil".

Este último punto nos hace plantear también una pregunta epistemológica del tipo ¿qué es primero? ¿El huevo o la gallina?

Si las conceptualizaciones y métodos provenientes desde la ciencia son capaces de alimentar las micro-culturas educativas organizando sus estructuras de sentido, y además, son capaces de establecer guías/patrones de comportamiento, vale la pena preguntarse si la investigación captura un fenómeno "natural" de ocurrencia en el contexto educativo o es que la investigación contribuye a su generación dentro de unos determinados marcos de comprensión. En otras palabras, habría que preguntarse si ¿el Compromiso Académico se refleja en la investigación o la investigación actual en la materia afecta la construcción del mismo?

En cualquier caso, analizar todos estos aspectos considerando como perspectiva un impacto positivo de la investigación sobre el Compromiso Académico en el desarrollo y educación de los estudiantes debe ser una materia activa en el campo de la educación.

\section{Conclusiones}

A través de los diferentes argumentos presentados es posible observar que, efectivamente, existen diferencias conceptuales y metodológicas entre las diversas aproximaciones al fenómeno del Compromiso Académico. 
Se observan dos principales formas en su abordaje con una base teórica fundamental común (TAD). A pesar de ello, se aprecian diferencias sustantivas entre los conceptos y modelos explicativos de ambas:

1. Uno de los enfoques está principalmente centrado en una explicación funcionalista del Compromiso Académico donde la orientación a la tarea cobra un realce sustantivo en la circunscripción del fenómeno.

2. En el otro enfoque se aprecia la influencia de aspectos contextuales, interaccionales y también individuales donde no queda clara la ponderación de cada uno de los distintos elementos. Sin embargo, este parece mayormente marcado por la influencia de factores externos frente a la dimensión interna de los individuos.

A través de los argumentos se observó que estas distintas aproximaciones, si bien tienen a la base una misma teoría explicativa de la conducta humana, involucran matices en la concepción sobre la educación y sus principales actores, que decantan directamente en posibilidades de abordaje diferentes del mismo fenómeno.

Es así que, en el enfoque JD-R el rol del estudiante es principalmente pasivo y el nivel de Compromiso Académico exhibido es un estado cognitivo-afectivo que ocurre como producto de una serie de variables circunscritas a la tensión entre las demandas y los recursos del estudiante para hacerles frente.

Desde el enfoque Tridimensional, por otro lado, se incluye una mayor participación del estudiante a partir de sus motivaciones en la toma de decisiones, pero estas también se encuentran circunscritas al plano específico del contexto educativo cotidiano e inmediato.

Basado en las razones anteriores, se realiza una crítica sobre el alcance de la TAD para la explicación del Compromiso Académico en base a las necesidades de Competencia, Relación y Autonomía, las cuales, parecen no ser suficientes para la explicación completa del fenómeno. Por lo anterior, se propone la falta de consideración de un rol trascendental del desarrollo de la identidad personal y profesional, así como la incorporación de las proyecciones futuras y las orientaciones altruistas de los estudiantes.

La observación de estos distintos aspectos permite analizar críticamente:

1. La posibilidad de que esté operando un enfoque top-down desde la construcción científica de la teoría, modelos teóricos y conceptos hacia la práctica cotidiana 
donde estos afecten los significados y marcos de funcionamiento que orientan el comportamiento en la Educación Superior. La pregunta que surge a partir de ello es ¿En qué medida podrían estar afectando las construcciones conceptuales en los estándares con los que se desarrolla regularmente la práctica?

2. Vinculado con lo anterior, la posible existencia de una retroalimentación bidireccional entre los conceptos construidos desde la ciencia y las prácticas ejercidas (a través de los significados, instituciones y herramientas). De este modo, otra pregunta de gran importancia que surge al respecto es ¿La investigación científica en el área está siendo capaz de capturar el fenómeno del Compromiso Académico o genera una forma de comprensión que lo define de forma sustantiva?

3. Las posibles consecuencias de entender el Compromiso Académico como se ha descrito en ambas aproximaciones. Una reflexión crítica de estas concepciones puede plantear dudas sobre cómo estos modelos podrían favorecer el rol pasivo del estudiante (tal vez favoreciendo un estilo de atribución causal y locus de control) y delimitarlo al contexto académico y cotidiano, además de incrementar la responsabilidad de los sistemas educativos sobre la producción de entornos que generan mayor Compromiso Académico.

\section{Bibliografía}

Appleton, J., Christenson, S., \& Furlong, M. (2008). Student Engagement with school: Critical conceptual and methodological issues of the construct. Psychology in the Schools, 45(5), 369-386. https://doi.org/10.1002/pits

Archambault, I., Janosz, M., Fallu, J.-S., \& Pagani, L. (2009). Student engagement and its relationship with early high school dropout. Journal of Adolescence, 32, 651670. https://doi.org/10.1016/j.adolescence.2008.06.007

Bakker, A., \& Demerouti, E. (2014). La teoría de las demandas y los recursos laborales. Journal of Work and Organizational Psychology, 29, 107-115. https://doi.org/10.1016/j.rpto.2017.07.003 
Bielefeldt, A., \& Canney, N. (2014). Impacts of Service-Learning on the Professional Social Responsibility Attitudes of Engineering Students. International Journal for Service Learning in Engineering, 9(2), 47-63.

Carvalho, V., De Oliveira, L., \& Pereira do Rêgo, D. (2010). Interacionismo simbólico: origens, pressupostos e contribuições aos estudos em Psicologia Social. Psicologia: Ciência E Profissão, 30(1), 146-161. https://doi.org/10.1590/S141498932010000100011

Casuso-holgado, M. J., Cuesta-vargas, A. I., Moreno-morales, N., Labajos-manzanares, M. T., Barón-lópez, F. J., \& Vega-cuesta, M. (2013). The association between academic engagement and achievement in health sciences students. BMC Medical Education, 13(33), 1-7.

Chang, M., Sharkness, J., Hurtado, S., \& Newman, C. (2014). What Matters in College for Retaining Aspiring Scientists and Engineers From Underrepresented Racial Groups. Journal of Research in Science Teaching, 51(5), 555-580. https://doi.org/10.1002/tea.21146

Cox, D. W., Bjornsen, A. L., \& Krieshok, T. S. (2015). Occupational Engagement and Academic Major Satisfaction: Vocational Identity's Mediating Role. The Career Development Quarterly, 64, 169-180. https://doi.org/10.1002/cdq.12049

Deci, E. L., Olafsen, A. H., \& Ryan, R. M. (2017). Self-Determination Theory in Work Organizations: The State of a Science. The Annual Review of Organizational Psychology and Organizational Behavior, 4, 19-43.

Ferreyra, M., Avitabile, C., Botero, J., Haimovich, F., \& Urzúa, S. (2017). At a Crossroads: Higher Education in Latin America and the Caribbean. World Bank Group. Washington: World Bank Group. https://doi.org/10.1596/978-1-4648-10145

Fredricks, J., Blumenfeld, P., \& Paris, A. (2004). School Engagement : Potential of the Concept, State of the Evidence. Review of Educational Research, 74(1), 59-109.

Fredricks, J., \& Mccolskey, W. (2012). The Measurement of Student Engagement: A Comparative Analysis of Various Methods and Studen Self-report Instruments. In C. Christenson, S, Reschly, A., Wylie (Ed.), Handbook of Research on Student Engagement. (pp. 763-782). New York: Springer-Verlag. https://doi.org/10.1007/978-1-4614-2018-7 
Gandhi, J., Maalouf, S., \& Patton, J. (2015). the Implementation of Service Learning Into Engineering Management \& Construction Management Courses. In Proceedings of the American Society for Engineering Management 2015 International Annual $\begin{array}{lllll}\text { Conference } & \text { (pp. 1-10). } & \text { Retrieved }\end{array}$ http://search.proquest.com/docview/1751245869?accountid=16788

Gómez, P., Pérez, C., Parra, P., Oritz, L., Matus, O., McColl, P., ... Meyer, A. (2015). Relación entre el bienestar y el rendimiento académico en alumnos de primer año de medicina. Revista de Médica de Chile, 143, 930-937.

Hu, S., \& McCormick, A. C. (2012). An Engagement-Based Student Typology and Its Relationship to College Outcomes. Research in Higher Education, 53, 738-754. https://doi.org/10.1007/s11162-012-9254-7

Knizley, A., \& Coleman, T. (2014). Implementing Service-Learning into an Introductory Mechanical Engineering Course. In 2014 ASEE Southeast Section Conference Implementing.

Markus, G., Howard, J., \& King, D. (1993). American Educational Research Association. Educational Research, 15(4), 410-419.

Navarro, G., Vaccarezza, G., González, M. G., \& Catalán, R. (2015). Construcción de conocimiento en educación superior. Educación de competencias genéricas en la Universidad de Concepción, CHile. (G. Navarro, Ed.). Concepción: Universidad de Concepción.

Pérez-villalobos, C., Bonnefoy-dibarrat, C., Cabrera-flores, A., \& Peine-grandón, S. (2012). Problemas de salud mental en alumnos universitarios de primer año de Concepción, Chile. Anales de Psicología, 28(3), 797-804.

Ramírez, A. (2015). El lenguaje en la revelación: Performatividad y pragmática. Theologica Xaveriana, 65(180), 301-325. https://doi.org/10.11144/javeriana.tx65180.1rpp

Rizo, M. (2015). Construcción de la realidad, Comunicación y vida cotidiana - Una aproximación a la obra de Thomas Luckmann. Intercom: Revista Brasileira de Ciências Da Comunicação, 38(2), 19-38. https://doi.org/10.1590/1809-5844201522 Ryan, R., \& Deci, E. (2000). Self-Determination Theory and the Facilitation of Intrinsic Motivation, Social Development, and Well-Being. American Psychologist, 55(1), 68-78. https://doi.org/10.1037110003-066X.55.1.68 
Salanova, M., Schaufeli, W., Martínez, I., \& Bresó, E. (2009). How obstacles and facilitators predict academic performance: The mediating role of study burnout and engagement. Anxiety, Stress \&Coping, $1, \quad 1-18$. https://doi.org/10.1080/10615800802609965

Schaufeli, W. (2013). What is engagement? In Employee Engagement in Theory and Practice. (pp. 1-37). London: Routledge. https://doi.org/10.15171/ijhpm.2015.12

Schaufeli, W., Martínez, I., Marques, A., Salanova, M., \& Bakker, A. (2002). Burnout and Engagemente in university studentes. A Cross-National Study. Journal of CrossCultural Psychology, 33(5), 464-481.

Schaufeli, W., Salanova, M., González-Romá, V., \& Bakker, A. (2002). The measurement of engagement and burnout: A two sample confirmatory factor analytic approach. Journal of Happiness Studies, 3, 71-92.

Skinner, E., Furrer, C., Marchand, G., \& Kindermann, T. (2008). Engagement and Disaffection in the Classroom: Part of a Larger Motivational Dynamic? Journal of Educational Psychology, 100(4), 765-781. https://doi.org/10.1037/a0012840

Veiga, F. H., Reeve, J., Wentzel, K., \& Robu, V. (2014). Assessing students' engagement: A review of instruments with psychometric Qualities. In Envolvimento dos alunos na escola: perspetivas internacionais da psicologia e educação. (pp. 38-57). Instituto de Educação da Universidade de Lisboa. Retrieved from http://repositorio.ul.pt/handle/10451/18036

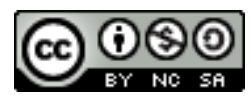

Esta obra está bajo una licencia de Creative Commons Reconocimiento-NoComercial-Compartirlgual 4.0 Internacional. 